\title{
Applications of Full-field Transmission X-ray Nanotomography and X-ray Nanospectroscopy at Stanford Synchrotron Radiation Lightsource
}

\author{
Simon Bare, Yijin Liu and Johanna Weker Nelson \\ SLAC National Accelerator Laboratory, Menlo Park, California, United States
}

There are many materials whose properties and performance are dependent on the local chemical and physical structure on a length scale in the tens of nanometer range. Such materials include catalysts, adsorbents and batteries. The development and application of nanoscale full-field X-ray tomography and X-ray absorption spectroscopy (XAS) has impacted our understanding of such materials. This talk will provide an introduction to full-field transmission X-ray nanospectroscopy and nanotomography, i.e. transmission X-ray microscopy (TXM), in 2D and 3D and will use specific examples from our research at Stanford Synchrotron Radiation Lightsource (SSRL) to illustrate the technique, the information content, and the new understanding that is gained from such studies.

The TXM located at SSRL is designed to work over an energy range of $\sim 5-14 \mathrm{keV}$. A capillary condenser focuses the beam to a spot of a few tens of micrometers on to the sample. The nominal spatial resolution of this system is $\approx 30 \mathrm{~nm}$. Typical exposure time for a single image is $\sim 0.5 \mathrm{~s}$. The system is fully automated for conducting complicated scans involving motions of the sample as well as all the optics in the system ${ }^{1}$.

The first case study is the investigation of the reaction kinetics in solid sorbent materials upon exposure to the reactive gas. For example, $\mathrm{CuO}$ is used to remove trace $\mathrm{H}_{2} \mathrm{~S}$ in natural gas purification. The aim of the study was to investigate whether heterogeneity at the particle or sub-particle scale affects the performance of the solid phase. First, XAS tracked changes in the average chemical environment of $\mathrm{Cu}$ atoms during sulfidation, and allowed determination of $\mathrm{CuO}$ conversion with time, providing direct determination of the bulk sulfidation kinetics. These data were then complemented by in-situ TXM that probed individual $\mathrm{mm}$ sized $\mathrm{CuO}$ particles to quantify chemical and structural changes on a length scale of tens of $\mathrm{nm}$. The data showed that sulfidation initiates on the outer portions of the particle and then propagates towards the center. Analysis of these data revealed similar kinetics of the sulfidation as those measured in a reactor containing many particles.

High Ni, low Co layered oxides are promising candidates as the cathode material with Li metal as the anode. Recent studies have discovered that engineering the grain crystallographic orientation could modulate the ion transport pathway in polycrystalline particles to enable rapid ion reactions ${ }^{2,3}$ \{Yoon, 2017 \#7 $\{$ Xu, 2020 \#2\}\{Yoon, 2017 \#7\}\{Yoon, 2017 \#7\}. We studied two Ni-rich Li layered oxides $\left(\mathrm{LiNi}_{\mathrm{X}} \mathrm{Mn}_{\mathrm{y}} \mathrm{Co} 1-\mathrm{x}-\mathrm{O} \mathrm{O}_{2}, \mathrm{NMC}\right)$ with similar redox active $\mathrm{Ni}$ content but with different grain crystallographic orientations, named rod-NMC with radially aligned grains, and gravel-NMC with randomly oriented grains $^{4}$. Gravel-NMC shows a lower retention of storage capacity on repeated cycles of charger and discharge. Using 3D TXM and nano-XAS at the Ni K-edge we show that the distinct charge distributions (Ni valence state distribution) in the two compositionally similar, yet, crystallographically dissimilar polycrystalline nickel-rich layered oxides (Figure 1). The three-dimensional charge mapping reveals that the internal charge distribution is guided by the crystallographic orientation of the constituent grains.

Full-field TXM also has applications in heterogeneous catalysis studies, for examples understanding catalyst deactivation in fluid catalytic cracking (FCC) catalysts. The FCC catalyst has a highly 
interconnected hierarchical pore-network in the catalyst. The pore space and its interconnectivity are of importance to the efficiency of the conversion process 5,6 .

In this work, we developed a correlative 3D micro-spectroscopic approach combining the methods of nano-TXM and multiple-element micro X-ray fluorescence (m-XRF) tomography ${ }^{7}$. On the basis of this correlative data set, we developed a detailed analysis approach studying the effect of metal deposition on the macro-pore network stability of a whole single FCC particle obtained from an industrial plant. Our approach allowed testing the response of the particle's pore network to a gradually increasing blockage of the pore channels by the poisoning metals. This effectively simulates catalytic ageing of the pore network that goes along with a decreasing cracking efficiency of the catalyst.

These examples highlight the broad application of full-field X-ray nano-X-ray absorption spectroscopy and tomography. There are many areas of science where information regarding the structure and chemical complexity at the tens of nm length scale is relevant to our understanding of such materials. Likewise there are continual technical developments in both the hardware (e.g. next generation synchrotron facilities, improved optics) and in our ability to utilize advanced algorithms in the data processing and data analysis (e.g. machine learning) that will present new opportunities for transmission X-ray microscopy.
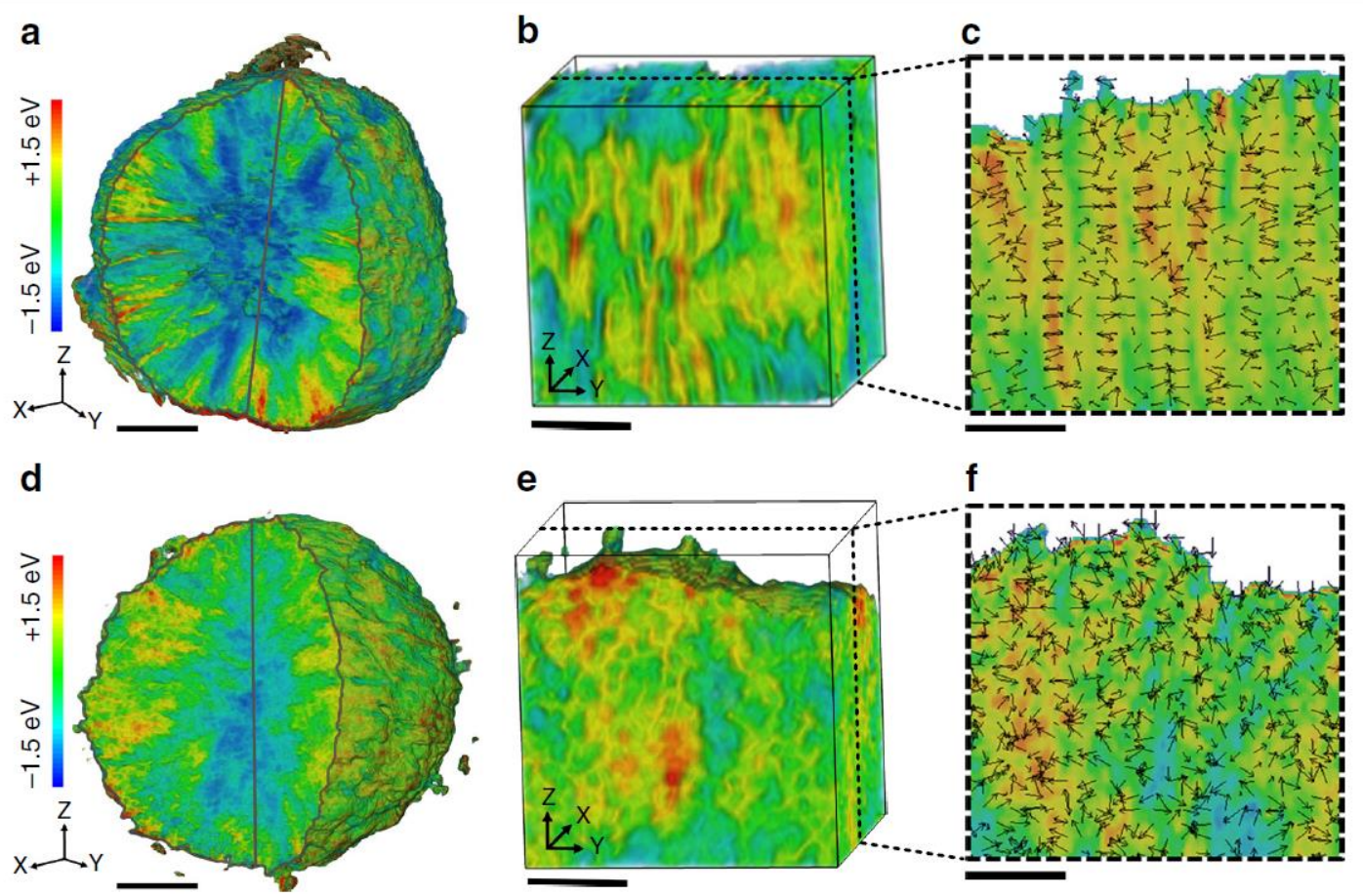

Figure 1. Charge distributions in the first charged state. (a) 3D Ni valance state distribution, (b) representative region of the 3D Ni valence state distribution and (c) 2D nanodomain valence gradient of the rod-NMC. (d) 3D Ni valence state distribution, (e) representative region of the 3D Ni valence state distribution and (f) 2D nanodomain valence gradient of the gravel-NMC. The scale bars in (a) and (d) are $3 \mu \mathrm{m}$, and in (b), (c), (e) and (f) $1 \mu \mathrm{m}$. The Ni K-edge absorption energies are color coded: blue $=$ lower energy, red $=$ higher edge energy. 
References

1. Liu, Y.; Meirer, F.; Wang, J.; Requena, G.; Williams, P.; Nelson, J.; Mehta, A.; Andrews, J. C.; Pianetta, P., 3D elemental sensitive imaging using transmission X-ray microscopy. Analytical and Bioanalytical Chemistry 2012, 404 (5), 1297-1301.

2. Yoon, C. S.; Park, K.-J.; Kim, U.-H.; Kang, K. H.; Ryu, H.-H.; Sun, Y.-K., High-Energy Ni-Rich $\mathrm{Li}[\mathrm{Nix} C o y M n 1-\mathrm{x}-\mathrm{y}] \mathrm{O} 2$ Cathodes via Compositional Partitioning for Next-Generation Electric Vehicles. Chemistry of Materials 2017, 29 (24), 10436-10445.

3. Liu, H.; Du, C.; Yin, G.; Song, B.; Zuo, P.; Cheng, X.; Ma, Y.; Gao, Y., An Li-rich oxide cathode material with mosaic spinel grain and a surface coating for high performance Li-ion batteries. Journal of Materials Chemistry A 2014, 2 (37), 15640-15646.

4. Xu, Z.; Jiang, Z.; Kuai, C.; Xu, R.; Qin, C.; Zhang, Y.; Rahman, M. M.; Wei, C.; Nordlund, D.; Sun, C.-J.; Xiao, X.; Du, X.-W.; Zhao, K.; Yan, P.; Liu, Y.; Lin, F., Charge distribution guided by grain crystallographic orientations in polycrystalline battery materials. Nature Communications 2020, 11 (1), 83.

5. Meirer, F.; Kalirai, S.; Morris, D.; Soparawalla, S.; Liu, Y.; Mesu, G.; Andrews, J. C.; Weckhuysen, B. M., Life and death of a single catalytic cracking particle. Science Advances 2015, 1 (3), e1400199.

6. Meirer, F.; Morris, D. T.; Kalirai, S.; Liu, Y.; Andrews, J. C.; Weckhuysen, B. M., Mapping Metals Incorporation of a Whole Single Catalyst Particle Using Element Specific X-ray Nanotomography. Journal of the American Chemical Society 2015, 137 (1), 102-105.

7. Liu, Y.; Meirer, F.; Krest, C. M.; Webb, S.; Weckhuysen, B. M., Relating structure and composition with accessibility of a single catalyst particle using correlative 3-dimensional micro-spectroscopy. Nature Communications 2016, 7 (1), 12634. 